\title{
Design of the optimal control for the active suspension
}

\author{
Sorin MARCU, Dinel POPA*, Nicolae-Doru STĂNESCU, \\ Nicolae PANDREA \\ University of Pitesti, Romania \\ *Corresponding author e-mail: dinel.popa@upit.ro
}

Article history

Received 01.11.2020

Accepted 15.02.2021

DOI https://doi.org/10.26825/bup.ar.2021.001

\begin{abstract}
The main purpose of the suspension is to minimize vertical acceleration. Through this paper we aim to analyze two PID and LQR control techniques to reduce system vibrations. The active system will be compared to a passive system using two types of profile. Matlab / Simulink software is used to evaluate the performance of the two controllers using a system with two degrees of freedom. The analysis shows that we can control the suspension system using the two techniques to improve the comfort and safety of the vehicle.
\end{abstract}

Keywords: passive suspension, active suspension, PID, LQR

\section{Introduction}

The suspension system is one of the important parts of the vehicle. When the damping coefficient is lower, comfort increases, but in this case, the stability is greatly reduced and vice versa.

Therefore, it is quite necessary to design a suspension system as optimal as possible to improve the comfort, maneuverability and stability of vehicles [1]. There are three basic types of suspension systems: passive, semi-active and active. Passive suspension is the least complex system and has many advantages. It consists of a shock absorber and a spring. The shock absorber acts as an energy dissipation element while the spring acts as an energy storage element [2]. But, the disadvantage of the passive suspension is that the performance is limited because the elements in its composition have fixed characteristics [3].

The semi-active suspension can be said to be a compromise between the passive and the active suspension system. Thus, in order to obtain better results, semi-active suspension systems use a variable spring and a shock absorber, which requires a small amount of external energy, the system is not very complex and can therefore provide significant progress [3].

The active suspension systems have the role of creating an energy source that generates the desired force in the system. In these systems, the developed force is produced directly by an electromechanical, pneumatic or hydraulic actuator, generally controlled by a computer that determines the required force in real time. The most important part of these systems is the development of a control strategy that optimizes the desired performance characteristics [4].

\section{Control methods}

The linear system. A continuous linear system has the model in shape:

$$
\begin{aligned}
& x(t)=A x(t)+B u(t) \\
& y(t)=C x(t)+D u(t)
\end{aligned} .
$$


Where $A$ is the matrix of state parameters, $B$ matrix of input parameters, $C$ matrix of output parameters and $D$ matrix of transmission parameters. For systems with constant parameters, the matrices $A, B, C$ and $D$ are constant while for systems with variable parameters at least one is depending on $t$.

The first equation of the system is the state equation, and the second the output equation. Because the equation of state is of the differential state type $x$ the state follows variations of the delayed input $u$.

In this paper, two types of controllers for the active suspension system are studied. These are PID (Proportional-Integral-Derivative) controllers and quadratic linear regulator (LQR).

PID controller. A typical structure of a PID control system is shown in Fig. 1, where it can be seen that in a PID controller, error signals $(t)$ error signals are used to generate proportional, integral and derived actions. The PID controller used in the active suspension study is a closed loop system. It aims to minimize error by changing control variables. For the best performance of the PID controller, its parameters must be adjusted according to the nature of the system.

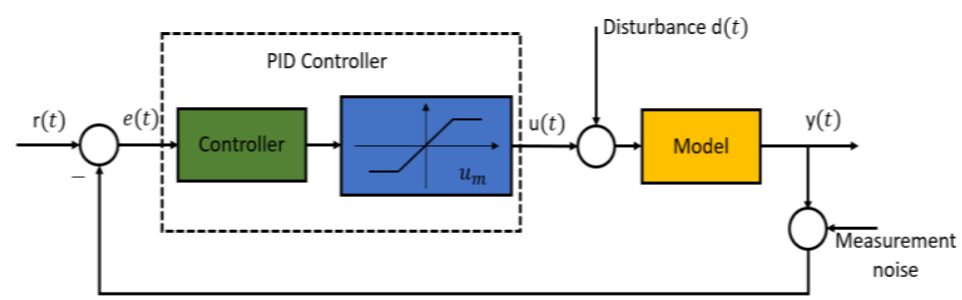

Figure 1. PID control structure.

A mathematical description of the PID controller is:

$$
u(t)=K_{p} e(t)+K_{i} \int_{0}^{t} e(t) d t+K_{d} \frac{d e(t)}{d t},
$$

where $u(t)$ is the model input signal, the error signal $e(t)$ is defined as, $e(t)=r(t)-y(t)$, and $r(t)$ is the reference signal, $K_{p}$ proportional controller, $K_{i}$ integral controller, $K_{d}$ derived controller.

A proportional controller $K_{p}$ will have the effect of reducing the rise time, integral controller $K_{i}$ will have the effect of eliminating the steady state error, but can change the transient response, and the derived controller $K_{d}$ will have the effect of increasing system stability and improving the transient response. The effects of each of the controllers $K_{p}, K_{i}$ and $K_{d}$ on a closed loop system are summarized in the table below.

\begin{tabular}{|c|c|c|c|c|}
\hline Answer & Rise time & Overshoot & Setting time & S-S Error \\
\hline$K_{p}$ & Decreases & Increase & Indefinite change & Decreases \\
\hline$K_{i}$ & Decreases & Increase & Increase & Discharge \\
\hline$K_{d}$ & Indefinite change & Decreases & Decreases & Indefinite change \\
\hline
\end{tabular}

LQR controller. LQR is a controller that gives an optimal response by minimizing the performance function, which refers to both state and input vectors.

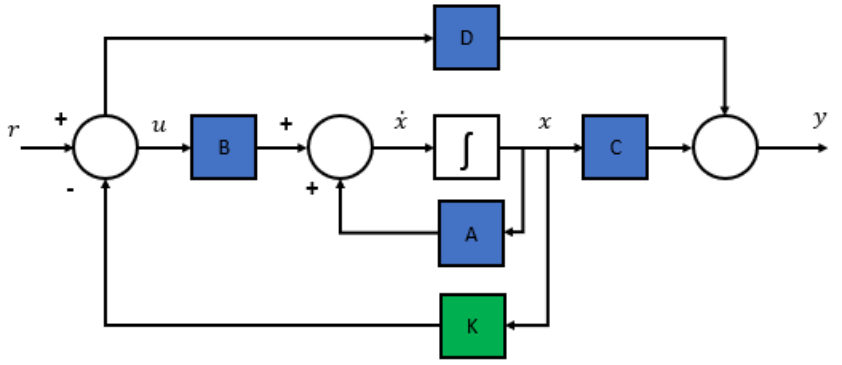


Figure 2. Closed loop Control system of LQR Controller The description of the LQR controller can be presented in the following form. Either the system $\dot{x}=A x+B u$ with the initial condition $x(0)$ is non-zero, it determines the input $u(t)$ input of the system by minimizing the performance function.

$$
J=\int_{0}^{\infty} x(t)^{T} Q x(t)+u(t)^{T} R u(t) d t .
$$

We assume that the linear system is stable, the optimal solution for the initial state is

$$
u(t)=-R^{-1} B^{T} P x(t)=-K x(t),
$$

where is the state reaction matrix, obtained using the following expression:

$$
K=-R^{-1} B^{T} P .
$$

$P$ being the solution of the Riccati equation

$$
A^{T} P+P A+Q-P B R^{-1} B^{T} P=0
$$

\section{Mathematical modeling}

The quarter car model is widely used in the dynamic analysis of cars for its simplicity, but also for the accuracy of the data obtained from the analysis at least in the initial design stage. Identifying the equations of motion for a suspension is done using Newton's second law.

Where: $M_{s}$ - sprung mass, $M_{u}$ - un-sprung mass, $K_{s}$ - spring stiffness coefficient, $K_{t}$ - tire stiffness coefficient, $C_{s}$ - damping coefficient, $F_{u}$ - force actuator, $Z_{r}$ - surface irregularities.

The equations of motion for passive suspension for both suspended and unsuspended mass are as follows. For the sprung mass $M_{s}$

$$
M_{s} \ddot{Z}_{s}=-C_{s}\left(\dot{Z}_{s}-\dot{Z}_{u}\right)-K_{s}\left(Z_{s}-Z_{u}\right) .
$$

For the unsprung mass $M_{u}$

$$
M_{u} \ddot{Z}_{u}=C_{s}\left(\dot{Z}_{s}-\dot{Z}_{u}\right)+K_{s}\left(Z_{s}-Z_{u}\right)-K_{t}\left(Z_{u}-Z_{r}\right) .
$$

These can be expressed as a matrix:

$$
\begin{aligned}
& {\left[\begin{array}{cc}
M_{s} & 0 \\
0 & M_{u}
\end{array}\right]\left\{\begin{array}{l}
\ddot{Z}_{s} \\
\ddot{Z}_{u}
\end{array}\right\}=\left[\begin{array}{cc}
-C_{s} & C_{s} \\
C_{s} & -C_{s}
\end{array}\right]\left\{\begin{array}{l}
\dot{Z}_{s} \\
\dot{Z}_{u}
\end{array}\right\}+\left[\begin{array}{cc}
-K_{s} & K_{s} \\
K_{s} & -\left(K_{s}+K_{t}\right)
\end{array}\right]\left\{\begin{array}{l}
Z_{s} \\
Z_{u}
\end{array}\right\}+\left\{\begin{array}{c}
0 \\
K_{t}
\end{array}\right\} Z_{r} .} \\
& \frac{1}{4} \text { of the } \\
& \text { vehicle mass } \\
& \text { Suspension } \\
& \text { system } \\
& \text { Wheel axle } \\
& \text { assembly } \\
& \text { Tire }
\end{aligned}
$$
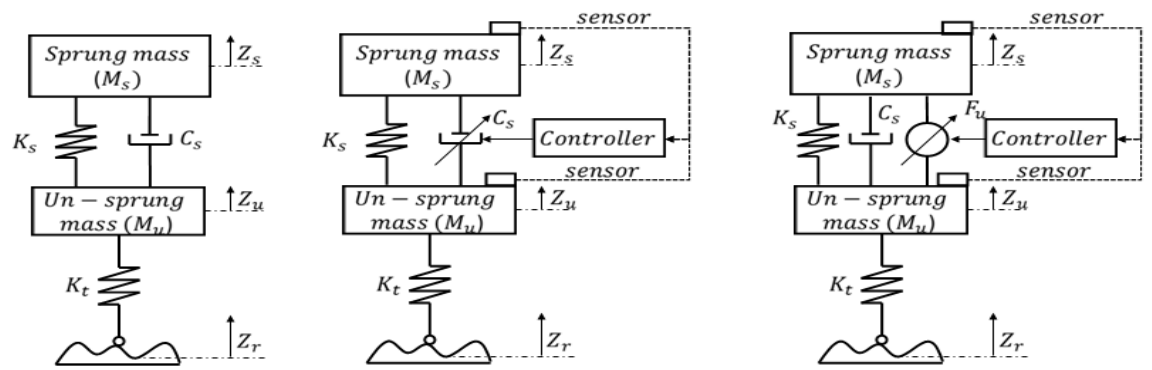


$$
M_{s} \ddot{Z}_{s}=C_{s}\left(\dot{Z}_{u}-\dot{Z}_{s}\right)+K_{s}\left(Z_{u}-Z_{s}\right)+F_{u} .
$$

For the unsprung mass $M_{u}$

$$
M_{u} \ddot{Z}_{u}=-C_{s}\left(\dot{Z}_{u}-\dot{Z}_{s}\right)-K_{s}\left(Z_{u}-Z_{s}\right)+K_{t}\left(Z_{r}-Z_{u}\right)-F_{u}
$$

Using notations $X_{1}=Z_{s}, \quad X_{2}=Z_{u}, X_{3}=\dot{Z}_{s}, \quad X_{4}=\dot{Z}_{u}$ the system results:

$$
\begin{aligned}
& \dot{X}_{1}=X_{3} \\
& \dot{X}_{2}=X_{4} \\
& \dot{X}_{3}=\frac{C_{s}}{M_{s}}\left(X_{4}-X_{3}\right)+\frac{K_{s}}{M_{s}}\left(X_{2}-X_{1}\right)+\frac{1}{M_{s}} F_{u} \\
& \dot{X}_{4}=-\frac{C_{s}}{M_{u}}\left(X_{4}-X_{3}\right)-\frac{K_{s}}{M_{u}}\left(X_{2}-X_{1}\right)+\frac{K_{t}}{M_{u}}\left(Z_{r}-X_{2}\right)-\frac{1}{M_{u}} F_{u}
\end{aligned}
$$

The matrix form is:

$$
\left[\begin{array}{l}
\dot{X}_{1} \\
\dot{X}_{2} \\
\dot{X}_{3} \\
\dot{X}_{4}
\end{array}\right]=\left[\begin{array}{cccc}
0 & 0 & 1 & 0 \\
0 & 0 & 0 & 1 \\
-\frac{K_{s}}{M_{s}} & \frac{K_{s}}{M_{s}} & -\frac{C_{s}}{M_{s}} & \frac{C_{s}}{M_{s}} \\
\frac{K_{s}}{M_{u}} & -\frac{K_{s}+K_{t}}{M_{u}} & \frac{C_{s}}{M_{u}} & -\frac{C_{s}}{M_{u}}
\end{array}\right]\left[\begin{array}{l}
X_{1} \\
X_{2} \\
X_{3} \\
X_{4}
\end{array}\right]+\left[\begin{array}{c}
0 \\
0 \\
\frac{1}{M_{s}} \\
-\frac{1}{M_{u}}
\end{array}\right]\left[F_{u}\right]+\left[\begin{array}{c}
0 \\
0 \\
0 \\
\frac{K_{t}}{M_{u}}
\end{array}\right]\left[Z_{r}\right] .
$$

This being a dynamic system of form

$$
\dot{X}=A X+B F_{u}+E Z_{r} \text {. }
$$

\section{Model simulation and results}

In this paper we will study two different types of profiles. The first is described by the mathematical equation and is represented in fig 4 . a.

$$
\text { road_profil_1 }=\left\{\begin{array}{cc}
a\{1-\cos (8 \pi t)\} & 2.5 \leq t \leq 2.75 \\
b(1-\cos (8 \pi t)) & 6.25 \leq t \leq 6.5, \\
0 & \text { otherwise }
\end{array}\right.
$$

where $\mathrm{a}=0,03$ and $\mathrm{b}=-0,01$.

The second profile is described by the mathematical equation and is represented in fig $4 . b$

$$
\text { road_profile_2 }=\left\{\begin{array}{cc}
c\{1-\cos (8 \pi t)\} & 2 \leq t \leq 2.25 \\
d\{1-\cos (8 \pi t)\} & 6 \leq t \leq 6.25, \\
0 & \text { otherwise }
\end{array}\right.
$$

where $\mathrm{c}=0,05$ and $\mathrm{d}=0,025$

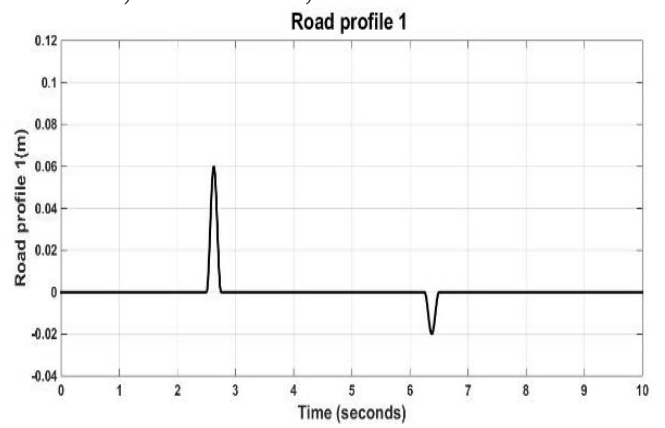

a) Road profile_1

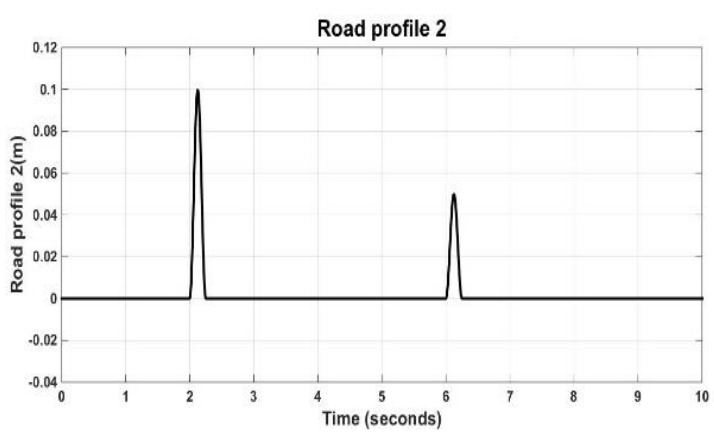

b)Road profile_2

Figure 4. Road profile. 
The values in the following table will be used to simulate the equations of motion.

Suspension parameters for simulation

\begin{tabular}{c|c|c|c}
\hline Suspension parameters & Sym & Unit & Value \\
\hline Sprung mass & $M_{s}$ & {$[\mathrm{~kg}]$} & 260 \\
\hline Un-sprung mass & $M_{u}$ & {$[\mathrm{~kg}]$} & 30 \\
\hline Damping coefficient & $C_{s}$ & {$\left[\mathrm{Nsm}^{-1}\right]$} & 1120 \\
\hline Spring stiffness coefficient & $K_{s}$ & {$\left[\mathrm{Nm}^{-1}\right]$} & 160000 \\
\hline Tire stiffness coefficient & $K_{t}$ & {$\left[\mathrm{Nm}^{-1}\right]$} & 210000
\end{tabular}

The results of the simulations for the two profile types are presented in the figures below.
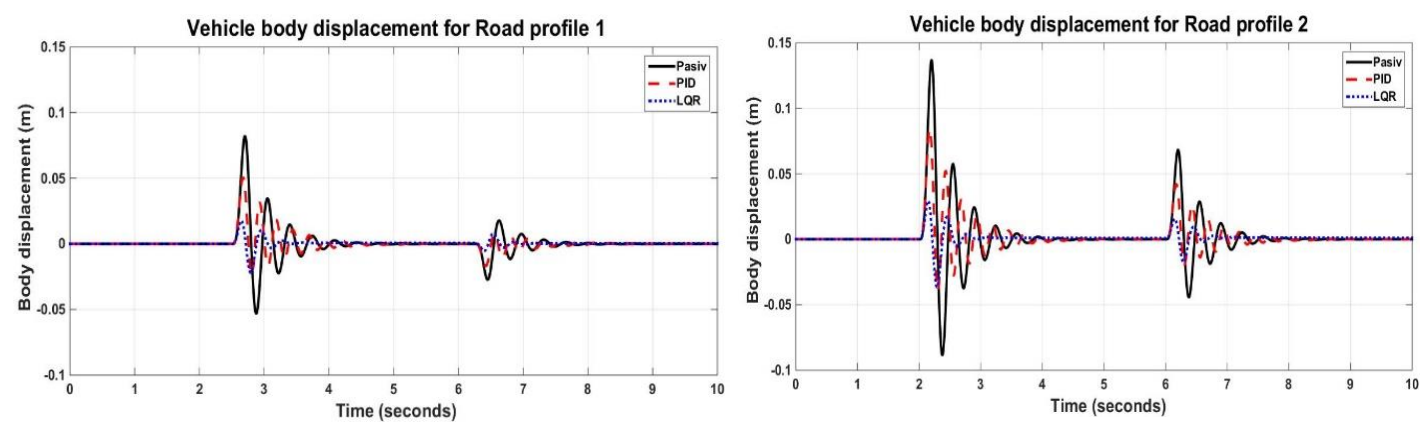

Figure 5. Vehicle body displacement.
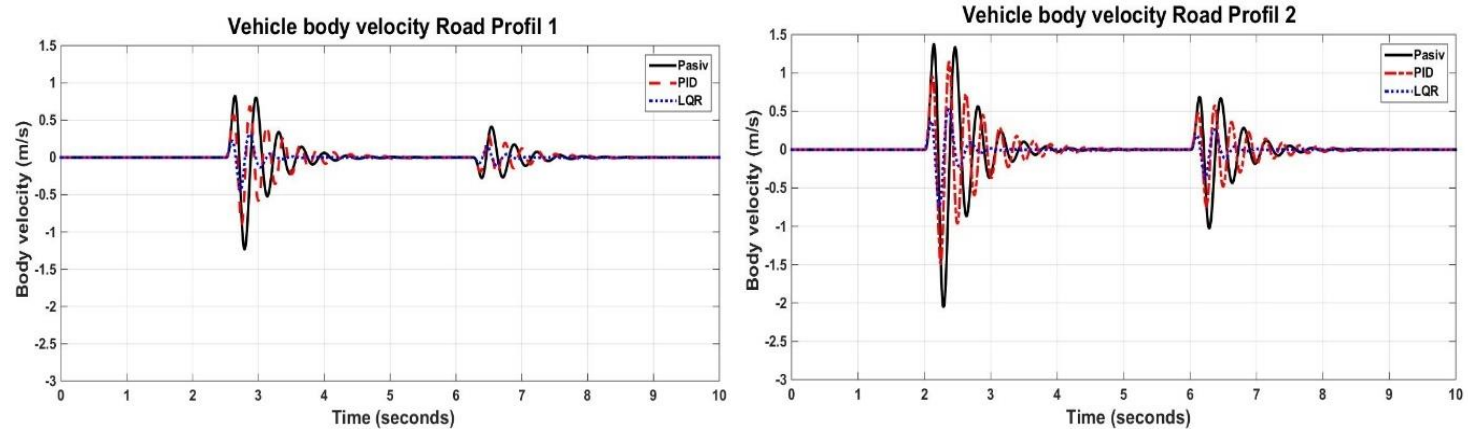

Figure 6. Vehicle body velocity

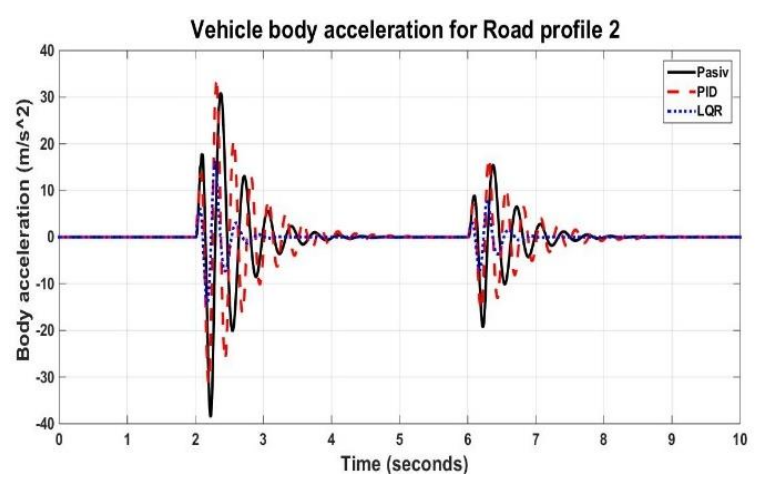

Figure 7. Vehicle body acceleration

The results of the simulation show that the active suspension provides high passenger comfort by reducing vibration compared to the passive suspension.. 


\section{Conclusions}

This paper described two PID and LQR control systems used in the control of a suspension system. PID and LQR controllers have been designed for high ride comfort, low amplitude for suspension travel and short stabilization time to quickly reach steady state.

To understand the efficiency of the two controllers, a simulation was performed using two types of profile and the quarter car model.

The simulation showed that the active suspension system has a much more efficient control over the displacement of the suspended mass using the two types of PID and LQR controllers compared to the passive system, because in the passive system $K_{s}$ și $C_{s}$ are constant and therefore it is difficult to achieve optimum vehicle comfort.

This can be seen from the graphs presented above. As a comparison between the two types of controllers the active system that uses LQR, offers a better result compared to the system that uses PID.

The simulation of the suspension system was performed using the Matlab/Simulink software.

\section{References}

[1]. Shital P. Chavan, Sanjay H. Sawant Analysis of Quarter Car Passive Suspension System with Non Linear Suspension Parameters IJSRD Vol. 2, Issue 05, 2014 | ISSN (online): 23210613.

[2]. Trupti P. Phalke, Anirban. C. Mitra Design and Analysis of Vehicle Suspension System International Engineering Research Journal Page No 165-172.

[3]. Yumna Shahid, Yumna Shahid Comparative Analysis of Different Model-Based Controllers Using Active Vehicle Suspension System Algorithms 2020, 13, 10.

[4]. Shital M. Pawar, A.A. Panchwadkar Estimation of State Variables of Active Suspension System using Kalman Filter International Journal of Current Engineering and Technology EISSN 2277 - 4106, P-ISSN 2347 - 5161.

[5]. Vivek Kumar Maurya, Narinder Singh Bhangal Optimal Control of Vehicle Active Suspension System Journal of Automation and Control Engineering Vol. 6, No. 1, June 2018,

[6]. Eshkabilov, S.: Modeling and simulation of non-linear and hysteresis behavior of magnetorheological damper in the example of quarter-car model.

[7]. Sayel, M.: Constructing Control System for Active Suspension System. Contemporary Engineering Sciences, Vol. 5, 2012, no. 4, 189 - 200.

[8]. Pandrea, N., Popa D., Parlac S, Modele pentru studiul vibratiilor automobilelor, Ed. TIPARG, Pitesti, 2001.

[9].Guido, K,: Adaptive Control of Mechatronic Vehicle Suspension Systems. 2011.

Rajesh, R.: Vehicle Dynamics and Control, 2012.

[10]. Kum-Gil, S., Min-Sang, S., Seung-Bok, C.: Performance evaluation of electronic control suspension featuring vehicle ER dampers. Meccanica (2013) 48:121-134. 